\title{
Los recovecos de la traducción: análisis de las diferentes versiones de una selección de poemas de Octavio Paz en Sendas de Oku
}

\author{
Luis MANuEl López Gómez \\ Universidad Autónoma de Madrid \\ luismanuel.lopez@predoc.uam.es
}

Resumen: El presente estudio compara las versiones de una selección de poemas de la primera y de la segunda edición de Sendas de Oku, traducción de Octavio Paz y Eikichi Hayashiya del diario poético de Matsuo Bashō Oku no hosomichi. Tiene como objetivo, además, demostrar la utilización de traducciones francesas e inglesas para la transformación que sucede entre los poemas de la primera versión y los de la segunda. Palabras clave: Octavio Paz, Sendas de Oku, traducción, poesía.

The artifices of translation: analysis of the different versions of a selection of poems in Octavio Paz's Sendas de Oku

Summary: This paper compares the different versions of a selection of poems from the first and the second edition of Sendas de Oku, a translation by Octavio Paz and Eikichi Hayashiya on Matsuo Bashō's poetic diary Oku no hosomichi. It also aims to demonstrate that Paz used several translations of the work in English and French to rewrite the poems of the first version into those of the second one.

Keywords: Octavio Paz, Sendas de Oku, translation, poetry. 


\section{Introducción}

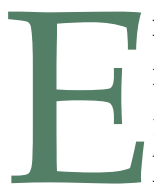

n 1957, Octavio Paz y su amigo Eikichi Hayashiya publican por primera vez en español la traducción del clásico japonés Oku no hosomichi, un diario poético de viaje escrito por el maestro renovador del haiku en el Japón premoderno: Matsuo Bashō. Trece años después, Octavio Paz convencerá a Eikichi Hayashiya de publicar una segunda versión del texto, más depurada: de los 65 poemas iniciales, 40 son reformados, ya sea parcial o totalmente. Esta reformulación textual parte de las lecturas de las distintas versiones inglesas y francesas del clásico que caen en manos de Paz y que el poeta mexicano utiliza para acceder por sí mismo al texto, ya que no hablaba japonés.

El número de publicaciones sobre la labor como traductor de haikus de Octavio Paz, si bien no es abundante, no es, ni mucho menos, escaso. Trabajos como los de Isnardi (1979) o Armand (1979) intuyeron acertadamente la capacidad de Paz para encontrar soluciones poéticas formidables a pesar de su desconocimiento de los idiomas originales de algunas obras literarias que tradujo del sánscrito, chino o japonés; otros, como Min (1979), explican inteligentemente la capacidad productiva del haiku que sobrevino precisamente por sus quehaceres de traducción. Y, sin embargo, a pesar de haber pasado cuarenta años de estas publicaciones, hoy día se sigue echando de menos trabajos que comparen los originales con sus traducciones, así como las fuentes que Paz utilizó para componer sus versiones. En el ámbito de la poesía japonesa, al menos, no parece que haya, todavía, ningún trabajo de este tipo -en lo que respecta a la poesía china, al menos, se atisba algunos trabajos que van más allá de la valoración general, como por ejemplo se puede ver en Botton (2011). Creo, por tanto, necesario abrir camino para un análisis crítico y filológico de las traducciones de Paz.

Con este trabajo, intentaré demostrar que dichas traducciones sirvieron a Paz para construir una versión de múltiples fuentes que le ayudaron a superar la restricción que suponía su falta de nociones del japonés. Para ello, me centraré en varios poemas que lo ejemplifican y prescindiré de la parte prosaica, ya que la prosa entre las dos versiones solamente sufre cambios para adquirir una mayor limpieza textual y es, en la práctica, el mismo texto que Hayashiya le dejó preparado: la labor de Octavio Paz con respecto a la 
prosa fue solamente la fijación del texto en un español más fluido y que la autoría de las notas al pie y del haibun pertenecen, en su mayoría, a Eikichi Hayashiya.

Así pues, este artículo está formado por tres partes: la descripción de las tres ediciones de Sendas de Oku y el contexto en que se produjeron, la relación de las traducciones que Paz utilizó como base y el análisis de una selección significativa de poemas. Cada uno de estos apartados arroja aspectos interesantes para reconsiderar el proceso de traducción de Octavio Paz como una vía entre la versión y la creación: en el primero, se explica la profunda revisión que el poeta mexicano emprende para las sucesivas ediciones de la traducción original; en el segundo, se da cuenta de las traducciones en otras lenguas que Paz utilizó para acceder por sí mismo al sentido de un texto cuyo idioma original no entendía; y, en el último, se analizan rigurosamente las traducciones de varios poemas teniendo en cuenta tanto su evolución con respecto a las versiones de la primera edición como sus semejanzas y diferencias con las fuentes en otros idiomas utilizadas por Paz. De esta manera, las dos primeras partes sirven como prolegómeno necesario para el análisis filológico aquí propuesto.

\section{Tres ediciones de Sendas de Oku}

La primera versión de Sendas de Oku vio la luz el 9 de abril de 1957, tres años después de que Octavio Paz y Eikichi Hayashiya se conocieran en el encargo de organizar una exposición de grabados japoneses: Octavio Paz era por entonces director general de organismos internacionales en la cancillería mexicana y Hayashiya acompañaba a la recién inaugurada embajada de Japón en México como agregado (Hayashiya, 2005: 85). Se produjeron 1000 ejemplares de la misma en la Imprenta universitaria de la Universidad Nacional Autónoma de México (UNAM), a cargo de Francisco González Guerrero, y no se realizó ninguna reimpresión, por lo que las posibilidades de acceso a su contenido son verdaderamente reducidas: solamente tengo constancia de dos ejemplares custodiados en la Biblioteca Central de la misma universidad.

La disposición del libro es simple y ordenada: una advertencia que sirve a modo de reclamo, dos páginas que hablan de la vida de Matsuo Bashō como 
presentación al público, un apartado sobre su poesía, la traducción en sí y el índice. Salvo algunos detalles que la deslucen, seguramente debidos a la dificultad en su revisión y a las prisas por la impresión, la traducción cumple efectivamente con su cometido: la prosa sigue fielmente al texto y los poemas son, más o menos, un reflejo fidedigno, más en el contenido que en la forma, de lo que son los haikus de Oku no hosomichi. Algunos topónimos y nombres propios, así como algunos vocablos mantenidos en japonés, aparecen mal escritos, cuestión que quizás se deba a un error de transcripción por parte de Paz. A todo el texto le acompaña un profuso cuerpo de notas al pie, que ayudan a entender detalles que el lector no podría reconocer leyendo solamente la crónica.

Diez años después de la primera publicación (1968) Hayashiya, que había desempeñado otros trabajos en Japón, regresó a México como consejero en la embajada. Al comienzo de la estancia, preguntó por Paz, que acababa de renunciar a su puesto como embajador en la India en protesta por la matanza de Tlatelolco, una masacre en la que centenares de personas pertenecientes al colectivo estudiantil fueron asesinadas por manos del ejército mexicano durante unas protestas en la Plaza de las Tres Culturas. No fue hasta un año y medio después (1970) cuando Paz envió una carta a Hayashiya desde Cambridge para comunicarle a su amigo el deseo de reformar la traducción iniciada quince años atrás e incluir las ilustraciones en tinta que Buson había hecho sobre Oku no hosomichi. Hayashiya mostró su beneplácito y le pidió que enviara la nueva versión para poder apreciarla. Esta no cambiaba nada del texto en prosa, pero casi la totalidad de los poemas sufrió una reformulación, además de que Paz introdujo una profusa introducción al haiku y cambiaba en buena medida el contenido de las notas a pie de página. Esa versión de 1970 es, al menos en lo que se refiere estrictamente al texto, la versión que leemos hoy día.

El libro soñado por Paz que había emprendido con Hayashiya no se materializó, sin embargo, hasta el año 1992: faltaban los dibujos de Buson sobre Oku no hosomichi. En 1985, Hayashiya visita el Museo Itsuo, situado en la ciudad de Ikeda, prefectura de Osaka, para solicitar el permiso para la utilización de los dibujos, que allí se encontraba. Fue, como me dijo en una reunión que pudimos mantener en noviembre de 2015, una operación muy delicada, ya que el proceso de escaneo, para el que se usaron las técnicas más avanzadas en aquellos años, necesitaba la delicadeza suficiente para tratar 
unas pinturas con más de doscientos años de antigüedad sin que esto supusiese una alteración traumática de la tinta. Después de la medición de la Fundación Japón, el Fondo Ohira, la Fundación Bancomer y el mismo Museo Itsuo, el texto de la segunda edición, al que acompañaban ahora los dibujos de Buson y el texto original en japonés en sus caligrafías, se editó en Shinto-Tsushinsha, una importante casa editorial japonesa con sede en Nagoya y Tokio. Como expresa Hayashiya, cuando se realizó la presentación en 1993 en el auditorio de la Fundación Bancomer, se había realizado así «el sueño y la promesa de treinta y cinco años atrás» (2005: 88).

\section{Fuentes de traducción}

Si la primera versión de Sendas de Oku fue, tal y como asegura Paz, la primera traducción completa del clásico Oku no hosomichi a una lengua occidental, en 1970, año en que Paz y Hayashiya prepararon su nueva versión, esta realidad había cambiado: si bien en español Sendas de Oku mantenía su hegemonía solitaria como única traducción del clásico, en otras lenguas se podía encontrar, por aquella época, hasta un total de cuatro versiones impresas, a las que Paz tuvo acceso:

Entre 1957 y 1970 han aparecido muchas traducciones de la obrita de Basho. Cuatro han llegado a mis ojos, tres en inglés y una en francés. Por cierto, cada una de ellas, ofrece una versión diferente del título: The Narrow Road to the Deep North; Back Roads to Far Towns; La sente étroite du bout-dumonde; y The Narrow Road through the Provinces (Bashō, 2005: 9-10).

Gracias a la nota que Paz adjunta, por petición de Hayashiya, al poema que empieza con «Tregua de vidrio» y las cartas intercambiadas con Donald Keene, sabemos, además, que Paz utilizó también la versión inédita del japonólogo estadounidense («Incluyo la copia de tu maravillosa traducción de Bashō [...] Me deprime compararla con texto español. Gracias otras vez -me ayudó mucho», Asiain, 2014: 270), por lo que podemos atestiguar que el poeta mexicano se valió de, por lo menos, seis fuentes diferentes, resumidas a continuación:

The narrow road to the deep north and other travel sketches: En 1966, Yuasa Nobuyuki consiguió lo que Paz y Hayashiya se habían propuesto diez años antes: una versión directa del clásico Oku no hosomichi adornado con los dibujos 
de Buson. La versión del profesor Yuasa es excelente por sus explicaciones minuciosas sobre el texto japonés y muchas de las conclusiones intelectuales de Paz sobre Oku no hosomichi se deben en su mayor parte a la formidable introducción de Yuasa a su texto.

The narrow road through the provinces: En 1969, Earl Miner, destacado estudioso estadounidense de la poesía japonesa, publica en The Regents of the University of California el libro Japanese Poetics Diaries, una selección de crónicas literarias traducidas al inglés que incluye el Tosa Nikki, el Izumi Shikibu Nikki, Gyoga Manroku y Oku no hosomichi, apareciendo este último bajo el título señalado en este epígrafe.

Back roads to far towns: Traducción de Cid Cormand y Kamaike Susumu en 1968. La versión es extremadamente literal y prefiere un estilo nominal que dificulta su lectura, por lo que es quizás uno de los textos más herméticos de este listado, si bien los traductores aportan un buen número de notas paratextuales.

The Penguin book of Japanese verse: Aunque estrictamente no se trata de una traducción de Oku no hosomichi, sino de una antología de poesía japonesa, este libro de Geoffrey Brownas y Anthony Thwaite, de 1966, recoge varios poemas del clásico japonés que Octavio Paz utiliza para sus recreaciones.

Le sente étroite du Bout-du-Monde: En la japonología francesa, René Sieffert sentó cátedra por sus traducciones directas de gran calidad: a él le debemos muchas de las traducciones españolas que, partiendo de sus textos en francés, presentaron en nuestro país las obras de, por ejemplo, Tanizaki. Aunque no sería hasta 1988 cuando Sieffert recogió los diarios poéticos completos de Bashō en Journaux de voyage, el traductor francés ya había publicado su versión de Oku no hosomichi en el número 6 de la revista L'ephémère en el año 1968. Sin lugar a duda, a Paz, al tanto de las publicaciones más importantes en literatura durante su época ${ }^{1}$, le debió de llamar la atención la traducción de Sieffert.

The narrow road to Oku: Este es, posiblemente, la fuente más complicada de valorar. Octavio Paz y Donald Keene habían trabado amistad en Nueva York

1 La revista L'Ephémère estaba dirigida por el renombrado escritor francés Yves Bonnefoy y acogió textos de Bataille, Pasternak y Beckett, entre muchos otros. 
en 1955 gracias a Faubion Bowers. En diciembre de ese mismo año, los dos intelectuales se reunieron nuevamente en Nueva York, reunión que el poeta mexicano aprovechó para recibir consejo de su amigo («El invierno pasado, en Nueva York, Donald Keene leyó nuestra traducción y me hizo algunas sugestiones que mejoraron nuestro texto. Doy aquí las más cumplidas gracias a tan generoso amigo», Bashō 2005: 34). Encontramos la misma información en los recuerdos de Keene:

Octavio Paz, que había terminado la traducción de Sendas de Oku con un amigo japonés y la comparaba con la mía, comentó que le gustaría traducir al español Sotoba Komachi, una pieza de Yukio Mishima que yo había traducido al inglés, para presentarla en México [...]

Paz me enviaba siempre sus libros (Mi español no es perfecto: lo estudié dos años en el bachillerato y llegué a hablarlo bastante bien, pero no lo he practicado durante mucho tiempo y no me expreso con confianza. Puedo leer sin problemas, sin embargo, siempre que no se trate de un diario, porque entonces necesito tener un diccionario al lado.) Yo también le enviaba los míos, y no solo los leyó todos, como era de esperarse, sino que llegó a citarme más de una vez, lo que desde luego fue un gran honor para mí (Asiain, 2014: 292).

La traducción completa de Keene no vería la luz hasta 1996, aunque una versión parcial fue publicada en 1955 en su Anthology of Japanese Poetry. Por motivos materiales, desconozco hasta qué punto se asemejan la versión inédita que manejó Paz con la publicación de ese mismo año. En cualquier caso, es evidente que sirvió para la comprensión del texto original y como base de la primera edición de Sendas de oku y que, además, siguió sirviendo como fuente fidedigna para las versiones posteriores de Paz.

\section{Análisis de los poemas}

De los cuarenta poemas reformados con respecto a la primera edición, se han escogido cinco de distintos pasajes de Sendas de Oku. La selección no es arbitraria: se trata de poemas con una reformulación significativa en los que se puede apreciar la indudable influencia de las traducciones en otros idiomas de las que se valió Octavio Paz. La muestra, por tanto, se configura 
como el máximo exponente del modo de proceder paciano en la traducción del clásico japonés y servir, además, como caso paradigmático del mismo. Todos los poemas originales en japonés están sacados de Bashō (2012), por lo que solo señalaré la página. Procedo de la misma manera con las traducciones de Paz: a la izquierda transcribo el poema de la primera versión (Bashō, 1957) y, a la derecha, los de la segunda (Bashō, 2005).

\subsection{Primer poema}

\begin{tabular}{|l|l|}
\hline $\begin{array}{l}\text { 草の戸も } \\
\text { 住替る代ぞ } \\
\text { ひなの家 (12) }\end{array}$ & $\begin{array}{l}\text { Kusa no to mo } \\
\text { Sumikawaru yo zo } \\
\text { Hina no ie }\end{array}$ \\
\hline $\begin{array}{l}\text { También esta cabaña de paja } \\
\text { en este mundo tornadizo } \\
\text { ha de transformarse en casa de muñecas (25) }\end{array}$ & $\begin{array}{l}\text { Otros ahora } \\
\text { En mi choza - mañana: } \\
\text { casa de muñecas. (67) }\end{array}$ \\
\hline
\end{tabular}

Este poema se inserta dentro del prólogo de Oku no hosomichi, justo en el momento en que Bashō abandona su choza para iniciar su viaje, de tal manera que preludia los caminos que va a transitar desde la visión opuesta de la morada abandonada por el poeta: en lugar del vacío, se presenta a una familia que mora en un futuro imaginario la vivienda. Hina no ie, «casa de muñecas», es una referencia a la festividad del día de las niñas, que las familias japonesas celebran adornando la entrada de sus casas con muñecas que representan la corte imperial del Japón medieval.

La primera versión de Paz es mucho más explicativa que la segunda, la cual es, por otra parte, mucho más sugerente. La expresión sumikawaru yo zo («en este mundo tornadizo» según Paz; literalmente, «las generaciones que se suceden y cambian $»^{2}$ ) se refiere a las nuevas generaciones que habitarán la casa, pero alude también al tiempo que pasa, expresado en forma de cambio. «En este mundo tornadizo» se desvía ligeramente de esa idea del tiempo cambiante cristalizada en unos nuevos moradores: Paz pone el foco en la totalidad del mundo, mientras que Bashō había elegido la concreción de la casa y su interior. La elección, sin embargo, parece ser hereda-

2 Cuando no se señala, la traducción es mía. 
da, ya que, como se puede apreciar, el poema es una traducción literal de la versión de Donald Keene:

\section{Even a thatched hut \\ in this changing world may turn \\ into a doll's house. (1955: 363)}

La segunda versión tiene un sabor más cercano al haiku, lo que se percibe, inicialmente, en su nueva forma: mantiene una versificación 5-7-6 que, si bien no es el esquema 5-7-5 del original, al menos es una escisión métrica muy utilizada en la composición de haiku (si el de Paz fuera un poema escrito en japonés, diríamos que el último verso es resultado de un caso de jiamari 字あまり, es decir, exceso de una mora). Por otra parte, mantiene la separación en dos partes del original e, incluso, hace una partición en términos bimembres (ahora-choza / mañana-casa de muñecas). Se sacrifica el motivo de la puerta de hierba para conseguir unicidad, aunque no es una pérdida relevante. La suplantación de sumikawaru yo zo por el término «otros» es una solución al exceso de sílabas formidable que parece haber sido tomada de la versión de Sieffert:

Ma chaumière même

d'autres a cette heure l'habitent

maison de poupées (2016: 73)

\subsection{Segundo poema}

\begin{tabular}{|l|l|}
\hline $\begin{array}{l}\text { 行く春や } \\
\text { 鳥啼き魚の } \\
\text { 目八泪 (13) }\end{array}$ & $\begin{array}{l}\text { Yuku haru ya } \\
\text { Tori naki uo no } \\
\text { Me wa namida }\end{array}$ \\
\hline $\begin{array}{l}\text { Pronto se va la primavera, } \\
\text { lloran los pájaros y hay lágrimas } \\
\text { en los ojos de los peces. (26) }\end{array}$ & $\begin{array}{l}\text { Se va la primavera, } \\
\text { quejas de pájaros, lágrimas } \\
\text { en los ojos de los peces. (69) }\end{array}$ \\
\hline
\end{tabular}

El poema expresa la pena por la despedida de Bashō de su hogar para iniciar el viaje alterando dos imágenes simbólicas que han sido utilizadas recurrentemente en la poesía clásica china y japonesa para conseguir tal efecto: el lamento del pájaro (del ánsar, concretamente) y del pez (Ebara, 1979: 42). 
El poema en sí no tiene una especial dificultad en la traducción de su contenido: cualquier lector de poesía es capaz de reconocer con asombro la elegancia y la tristeza contenida en las lágrimas de los peces disimuladas en el agua del río, incluso aunque no distinga el uso innovador que el poeta hace del imaginario clásico. No sucede lo mismo con la traducción de la forma: es una composición que mantiene con una armonía especial del esquema 5-7-5 del haiku, pero resulta complejo mantener el encadenamiento de elementos respetando el cómputo silábico en una lengua que se apoya en la explicación verbal como es el español. A esto se le añade otra dificultad: los versos están perfectamente ligados en más de dos niveles. Así, el verso primero (yuku haru ya «la primavera que se va») es el kigo, la palabra que indica la estación en que se escribe el haiku, y la síntesis del poema, pero el paisaje que describe se une, a su vez, a la emoción del verso final (me wa namida «los ojos [de los peces] son lágrimas»); tori naki («los pájaros cantan») y uo no («de los peces») forman un solo verso, pero están separados en dos planos por una cesura sintáctica, como si fueran dos realidades complementarias que ejemplifican la tristeza de la partida. Finalmente, el último verso se extiende a todo el poema, pero es un atributo directo de la segunda parte del verso ([...] uo no / me wa namida). Para expresar todo esto, el traductor debe optar, por tanto, por una versificación distinta a la métrica japonesa, es decir, más extensa.

En la primera versión, Paz parece ser consciente de esto. Al igual que en numerosos poemas de la primera edición, prefiere la extensión a la concreción. La influencia de la lectura de los clásicos japoneses en inglés fue, en mi opinión, determinante, ya que, en aquellos años, muchos traductores angloparlantes deciden extender los poemas explicativamente para un público poco acostumbrado a la forma densa del haiku e irremediablemente desconocedor de muchos aspectos que a un público japonés le puede resultar obvio. Al investigar un poco las fuentes de Paz, encontramos que su primera versión, de hecho, toma algunas de las aclaraciones como el pronto que acompaña a la primavera en el primer verso o el verbo haber para unir las lágrimas con los peces de la traducción que le envió Keene desde Nueva York:

Spring soon ends -

birds will weep while in

the eyes of fish are tears. (1955: 364) 
La segunda versión es más pulida y destierra los verbos en beneficio de un nominalismo más cercano al original. La separación por comas resta la excesiva unidad de los tres elementos, equiparados antes en un mismo plano al tratarse de una mera enumeración, y se consigue, por medio de la cesura, que se intensifica tanto por la repetición del esquema morfológico (nombre-nombre, quejas-lágrimas) como por la distancia acentual que se consigue con la reiteración de las esdrújulas (pájaros-lágrimas). El esquema métrico es un 7-8-8, aunque los versos están escanciados de tal manera que una lectura de 7-5-11 también sea posible e, incluso, más atractiva. Si bien el esquema no responde al del haiku, es una escansión totalmente lícita en cuanto a las posibilidades de la tradición poética hispánica.

\subsection{Tercer poema}

\begin{tabular}{|l|l|}
\hline $\begin{array}{l}\text { あらたふと } \\
\text { 青葉若葉の } \\
\text { 日の光 (16) }\end{array}$ & $\begin{array}{l}\text { Ara tōto } \\
\text { Aoba wakaba no } \\
\text { Hi no hikari }\end{array}$ \\
\hline $\begin{array}{l}\text { ¿Qué gloria! } \\
\text { Las hojas verdes, las hojas jóvenes, } \\
\text { bajo la luz del sol (29) }\end{array}$ & $\begin{array}{l}\text { Mirar, admirar } \\
\text { Hojas verdes, hojas nacientes } \\
\text { entre la luz solar (77) }\end{array}$ \\
\hline
\end{tabular}

El poema comporta la primera composición de verano de Bashō en el diario y supone una demarcación tajante que expresa la inmersión completa en el viaje preludiado de los capítulos anteriores.

Las dos versiones de Paz no son diametralmente opuestas entre sí, pero las diferencias, aunque sutiles, las separan. Ara tōto es una expresión de difícil traducción que alude a la sorpresa por la majestuosidad o la nobleza de algo. «QQué gloria!» es una buena forma de traducirlo, aunque el verso, además de ser algo corto, peca de ser algo banal, ya que se puede interpretar no en el sentido de glorioso, sino de sensación agradable o comodidad. El primer verso de la segunda versión es más cercano a la poesía de Paz: se intuye un sujeto que recomienda con su acción la capacidad liberadora de la contemplación y que ejemplifica, en su gesto, esa habilidad humana. Mirar es admirar, es decir, del gesto mundano y terrenal de la mirada nace la profundidad metafísica de la contemplación y, con ello, la integración y desintegración 
del sujeto en sí mismo y en el paisaje observado. En la mirada somos silencio y en la admiración gritamos silenciosamente. A diferencia de Wittgenstein, Paz cree en la capacidad expresiva del silencio y en la facultad del ser humano de sublimarse en dicho silencio.

El segundo verso de la versión primera es más fiel al original: wakaba son hojas jóvenes o, a lo sumo, hojas tiernas o frescas. «Hojas verdes, hojas nacientes» no acaba de expresar el verdor de la hoja ya tierna y plena, pero tiene su razón de ser: Paz se detiene no en la plenitud de la aparición del verano, sino en su condición de epifanía, de nacimiento admirado por el poeta que mira. Por ello, decide seguir a Sieffert en ese segundo verso (aunque, en el caso del traductor francés, el término naissante, que no se corresponde con la contemplación silenciosa sino con la exclamación, cambia el sentido):

\section{Ah pure merveille \\ feuille verte feuille naissante \\ au soleil que brille (2016: 75)}

El cambio en el tercer verso es, al mismo tiempo, un acierto y un desacierto. El acierto viene de la proposición: entre unifica mejor las hojas y el sol que bajo, que impone una jerarquía ${ }^{3}$. La luz solar, sin embargo, pierde un poco de fuerza frente a la luz del sol: el carácter 日 (sol) y el carácter 光 (luz) son separados en el original por la preposición de (no の), y digo son separados porque, juntos, aluden al topónimo en el que se escribe el poema: Nikkō 日 光. Es deseable, por tanto, mantener la confrontación de los dos sustantivos a acercarlos por medio de la adjetivación.

\footnotetext{
3 Personalmente, prefiero traducir el poema con la preposición en. Al leer el poema, la impresión que deja es la de una luz que atraviesa el cuerpo de las hojas, cuyas hebras se traslucen sin perder su forma. La luz verdosa y la hoja iluminada son las dos caras de la misma realidad y, de hecho, así lo expresa la preposición de del poema, que dice, literalmente, «la luz del sol de las hojas verdes y jóvenes»; son las hojas y el sol las que se funden en una sola luz.
} 


\subsection{Cuarto poema}

\begin{tabular}{|l|l|}
\hline $\begin{array}{l}\text { 松嶋や } \\
\text { 鶴に身をかれ } \\
\text { ほと〉ぎす } \\
\text { 筸良 (34) }\end{array}$ & $\begin{array}{l}\text { Matsushima ya } \\
\text { Tsuru ni mi wo kare } \\
\text { Hototogisu } \\
\text { Sora }\end{array}$ \\
\hline $\begin{array}{l}\text { En Matsushima } \\
\text { ipídele su plumaje a la grulla } \\
\text { oh ruiseñor! (54) }\end{array}$ & $\begin{array}{l}\text { En Matsushima } \\
\text { isus alas plata pídele, } \\
\text { tordo, a la grulla! (121) }\end{array}$ \\
\hline
\end{tabular}

El poema está compuesto por Sora, el discípulo que acompaña a Bashō en su viaje por Japón. El mismo poema se encuentra en Sarumino, una antología publicada por Bashō y sus discípulos Mukai Kyorai y Nozawa Bonchō en 1691, precedido por una frase que explica su sentido poético final: «Cuando fuimos a ver Matsushima, leí [unos versos que decían]: "toma prestado el chorlito el plumaje de la grulla"» (Ebara, 1941). En cuanto a su sentido estricto, Sora debió de oír el canto del cuclillo, pero pensó que, por muy hermoso que este fuera, su fragilidad no armonizaba con el paisaje poderoso y majestuoso de Matsushima, uno de los más célebres de Japón; la grulla, un ave más fuerte y celebrada por su longevidad, es más apropiada para la belleza eterna de Matsushima. No es un poema muy inspirado, aunque la hermosura de Matsushima es difícil de atrapar por la palabra; Bashō, consciente de esa dificultad, calla, al igual que en otras ocasiones, cuando el paisaje lo abruma y deja que sea su discípulo el que resuma la experiencia con sus versos.

Paz, en la segunda versión, parece haberse dado cuenta de la necesidad de enfatizar la fragilidad del cuco (pues ese es el ave llamada hototogisu, y no tordo ni ruiseñor) y la majestuosidad de la grulla. Para ello, cambia dos elementos troncales del poema: el ruiseñor pasa a ser un tordo y el plumaje de la grulla unas alas de plata. Nótese que, aunque es cuestionable la decisión de cambiar el nombre del pájaro, el motivo de la misma no es arbitrario: el ruiseñor, que ha sido muy bien tratado por la tradición literaria europea, podría haber competido con la grulla; el tordo, aunque tiene uno de los cantos más bellos de América, es relativamente un competidor menos contundente. La elección de Paz es, además, consciente, ya que, aunque todas las versiones 
incluyen como traducción al cuco, Paz elige la única que apuesta por un ave distinta, la de Earl Miner:

Here at Matsushima

O Wood thrush, the plumage of cranes

Would add to your song. (1969: 174)

Lo mismo ocurre con «las alas de plata» de las que Paz se apropia: el atributo, fabulación de Yuasa, no se encuentra en ninguna otra traducción y, sin embargo, la imagen ayuda a imaginar el vuelo de la grulla cortando con el poderío de sus alas blancas, plateadas, el sonido lastimero del cuco:

Clear voice cuckoo,

Even you will need

The silver wings of a crane

To span the islands of Matsushima (Bashō, 1966:116)

\subsection{Quinto poema}

\begin{tabular}{|l|l|}
\hline $\begin{array}{l}\text { 笈も太刀も } \\
\text { 五月にかざれ } \\
\text { 帋幟 (26) }\end{array}$ & $\begin{array}{l}\text { Oi mo tachi mo } \\
\text { Satsuki ni kazare } \\
\text { Kaminobori }\end{array}$ \\
\hline $\begin{array}{l}\text { Mochila y espada: } \\
\text { sacadlas en mayo } \\
\text { con los estandartes. (43) }\end{array}$ & $\begin{array}{l}\text { Espada y morral: } \\
\text { fiesta de muchachos, } \\
\text { banderas de papel... }\end{array}$ \\
\hline
\end{tabular}

El poema se refiere a las banderas con forma de carpa que se utilizan para celebrar el día del niño. La segunda versión está basada inequívocamente en la de Sieffert, de la que toma parte del tercer verso:

Hotte et sabre
exposé pour la cinquième lune
étendard de papier. (Bashō, 2016: 81)

Aunque la primera versión es más fiel a la presentación del original, ya que es una traducción casi literal, la segunda consigue transmitir con mayor eficacia la prisa de Bashō en el momento de escribir el poema. La espada y el 
morral hacen referencia al sable de Yoshitsune y al cofre de Benkei ${ }^{4}$, guardados como tesoros en el templo de Iōji. La idea de Bashō de mandar que adornen con esos objetos el festival de los niños no es más que una excusa para traer a colación esa celebración típica de mayo; el poema es, desde luego, un apunte rápido que no acaba de convencer como haiku en sí. La segunda versión de Paz, en el que se asocia vagamente la espada y el morral con las banderas de papel, casi como si fuera un pensamiento fugaz del poeta que esboza su poema, responden mejor al aire de improvisación que desprende la composición endeble de Bashō. Por otra parte, la relación traída por los pelos no es lo único que hacen pensar que Bashō no dedicó mucho tiempo a la revisión del haiku: aunque no se puede considerar un error, pues es algo común, el primer verso cuenta con una sílaba de más.

\subsection{Sexto poema}

\begin{tabular}{|l|l|}
\hline $\begin{array}{l}\text { 五月雨を } \\
\text { あつめて早し } \\
\text { 最上川 (42) }\end{array}$ & $\begin{array}{l}\text { Samidare wo } \\
\text { Atsumete hayashi } \\
\text { Mogamigawa }\end{array}$ \\
\hline $\begin{array}{l}\text { Junta todas las lluvias de mayo } \\
\text { y se lanza, rápido, } \\
\text { el río Mogami. (65) }\end{array}$ & $\begin{array}{l}\text { Junta las lluvias } \\
\text { del Quinto Mes el río - } \\
\text { y al mar las lanza. }\end{array}$ \\
\hline
\end{tabular}

Junto con muchos de los otros poemas de Oku no hosomichi, este es uno de los más conocidos y memorizados por los japoneses de toda la obra de Bashō. Compuesto el día 23 del quinto mes del calendario lunar, el haiku encabezó una sesión poética en la casa de Takano Ichiei, en Ōishida. Bashō, cuando incorporó el poema a Oku no hosomichi, apenas introdujo un cambio de un adjetivo (suzushi 涼し, «fresco» por hayashi 早し, «rápido»). Durante el quinto mes del calendario lunar, entre junio y julio, al archipiélago japonés llega un frente de lluvias conocido como tsuyu 梅雨, «la lluvia del ciruelo». Es uno de los períodos con más humedad del año en Japón, y la lluvia, aunque no suele caer en ráfagas, no cesa en ningún momento. Bashō, impresionado por la rapidez de las aguas del río Mo-

4 Minamoto no Yoshitsune, famoso general japonés del siglo XII, y su vasallo. Juntos ocupan un lugar destacado en la historia y en el de Japón. 
gamigawa (es uno de los tres ríos con la corriente más rápida del país), compara su velocidad con su capacidad de devorar las lluvias que caen por el tsuyu: «Recoge las aguas de Samidare, [la lluvia de mayo], veloz, el río Mogamigawa».

La versión primera de Paz es correcta y viene a expresar casi exactamente lo mismo que el original. La segunda versión, sin embargo, es más problemática. Quizás consciente de la fama de los versos, Paz parece desear hacer del poema un haiku también métricamente. Esto, a nivel de traducción, pasa a ser, sin embargo, un empobrecimiento del poema por los siguientes dos motivos.

El primero de ellos es la supresión del topónimo. Aunque se hable del río Mogamigawa en la parte prosaica del diario, el haiku necesita mantener siempre una independencia estético-semántica: el haibun, la creación de un texto que explica un poema y de un poema que embellezca el texto, no puede eliminar, por muy interrelacionados que estén, la autonomía estética de cada una de las partes, es decir: en un haibun sus partes están relacionadas pero no son interdependientes. Al eliminar la alusión al río Mogamigawa, el haiku pierde su capacidad de expresar la realidad del río poéticamente y el lector que quiera entender el texto deberá recurrir al fragmento anterior. El río que nos presenta Octavio Paz puede ser cualquiera y, desgraciadamente, debemos encontrar la respuesta en el párrafo anterior en prosa que le precede. Es más, el Mogamigawa es el elemento de sorpresa que da sentido al poema en la estructura casi de adivinanza de la que gustan una gran mayoría de los haikus: la lluvia cae en el primer verso y el lector, en apenas una décima de segunda, se extraña de que se la recoja con gran velocidad; al extrañamiento le llega, después, la revelación: es el agua velocísima del río Mogamigawa. Por si fuera poco, ninguno de los otros traductores que Paz sigue (Yuasa, Miner, Keene y Sieffert) olvida la importancia del topónimo en el poema5:

Gathering all the rains

Of May

The river Mogami rushes down

In one violent stream (Bashō, 1966: 124)

5 Nótese, además, que, salvo la versión de Yuasa, todos colocan el topónimo en el verso final para dar el susodicho efecto de sorpresa. 
The summer rains

Collected in a surging current:

The Mogami River (Miner, 1969: 181)

Gathering seawards

The summer rains, how swift it is!

Mogami River (Bashō, 1999:100)

Grosse de pluies

de saison rapide court

la Mogami-gawa (Bashō, 2016: 92)

El segundo es un añadido poético que Bashō no había pensado. Si el poema hubiese acabado en un «Mogamigawa $»^{6}$, la traducción sería perfecta. Pero Paz, que conscientemente no quería incluir el topónimo porque sería un sinónimo del «río» del segundo verso, decide, agotados todos los versos del original, rematarlo todo con un «al mar las lanza». La ocurrencia debía de venir, sin duda, como paralelismo del poema 38, en el que el río Mogamigawa hunde al sol en el mar. La idea, aunque pertinente, para Bashō sería impensable, porque diferiría mucho con lo que él veía: desde Ōishida, es imposible ver la desembocadura del Mogamigawa, situada a una distancia de más de $76 \mathrm{~km}$. El poema es, por tanto, una manipulación consciente de Paz para enlazarlo con el poema 38 y crear un nexo de significado nuevo.

\section{Conclusiones}

Octavio Paz no se conformó con las sugerencias de su compañero de traducción y decidió, por su propia cuenta, acceder a un texto para el que le faltaban los medios. Lector voraz antes que poeta, la labor de Paz con respecto a la segunda edición de su traducción demuestra la capacidad de investigación filológica del escritor mexicano. Como si de un puzle se tratara, adelantándose varios años a la ecdótica francesa, Paz recompone el sentido original del texto buceando entre los juegos y las licencias de todos los traductores que se acercaron a Oku no hosomichi entre los años cincuenta y los sesenta. La muestra de poemas confirma la utilización sistemática de las fuentes francesas e inglesas que Paz conocía en el momento

6 -gawa significa río. Por lo general, no se suele traducir y se interpreta como un topónimo. 
de recomponer su primera versión de Oku no hosomichi. No solamente se vale de estos textos para entender el poema, sino que, además, los combina y reforma a su voluntad para conseguir una versión literaria más potente y atractiva; en ocasiones, incluso, los ignora, sin refutarlos, para poder llegar a una realidad poética distinta, tal y como ya apreció Hayashiya en sus discusiones con su amigo respecto a sus versiones: «[...] sentí una gran emoción, casi un estremecimiento, cuando caí en cuenta de la forma en que Octavio Luchaba para traducir un poema de Bashō, cavilando y buscando una palabra a fin de transmitir el sentido real» (2005:88). Octavio Paz no pudo refrenar su sentir de poeta, e imaginando ser Bashō, escribió las versiones de unos poemas que, paradójicamente, pueden sentirse como los más atrevidos o los más fieles: una profanidad a ojos del traductor o una aceptación del espíritu de la poesía que, al saltar a otra lengua, se convierte en un eco de su sentido.

Por motivos de espacio y de forma, el análisis completo de todos los poemas de Sendas de Oku no tiene cabida en este artículo. Sin embargo, los poemas aquí señalados pueden ser tomados como una prueba irrefutable de la refundición paciana y servir de punto de partida para un análisis mayor que englobe la totalidad de poemas de su traducción del clásico japonés. Asimismo, se abre una línea interesante a tomar en cuenta en futuras investigaciones: la comparación entre las traducciones de Octavio Paz, los originales traducidos y su poesía. Al fin y al cabo, se trata de un campo poco transitado en español, y Octavio Paz tradujo directa o indirectamente de un gran número de literaturas, algunas de lenguas poco habladas en España (húngaro, sánscrito), otras un poco más conocidas (chino, idioma que maneja una generación de hispanistas bilingües desde hace relativamente poco) y un buen grueso de poemas escritos en idiomas con el que se ha tenido contacto a lo largo de toda la historia de la literatura en español (portugués, inglés y francés). Sería interesante, por tanto, descubrir en qué medida asimiló Octavio Paz dichos poemas y sus tradiciones y arrojar un poco más de luz a la obra de un poeta que sin dejar de ser mexicano fue decididamente universal. 


\section{Referencias bibliográficas}

Armand, Octavio (1979), «Octavio Paz o el traductor no traiciona», en Cuadernos Hispanoamericanos, 343-345. págs. 732-738. En línea: http://www. bibliotecanacionaldigital.gob.cl/visor/BND:343113.Último acceso el 12 de junio de 2020.

Asiain, Aurelio (2014), Japón en Octavio Paz, México, Fondo de Cultura Económica.

BASHō (1957), Sendas de Oku, traducción de Octavio Paz y Eikichi Hayashiya, México, Universidad Nacional Autónoma de México

- (1966), The narrow road to the Deep North and other travel sketches, traducción de Nobuyuki Yuasa, Bungay, Penguin Books

- (1970), Sendas de Oku, traducción de Octavio Paz y Eikichi Hayashiya, Barcelona, Barral.

- (1996a) Back roads to far towns, traducción de Cid Corman y Kamaike Susumu, Nueva Jersey, The Ecco Press

- (1996b), The narrow road to Oku, traducción de Donald Keene, Tokio, Kodansha International.

- (2005), Sendas de Oku, traducción de Octavio Paz y Eikichi Hayashiya, México, Fondo de Cultura Económica.

- (2012), Oku no hosomichi, edición de Tsutomu Ogata, Tokio, Kadokawa Sophia Bunko.

- (2016), Journaux de voyage, traducción de René Sieffert, Lonrai, Verdier.

Bownas, Geoffrey y ThwaIte, Anthony (1968) [1964], The Penguin Book of Japanese Verse, Bungay, Penguin Books.

Ebara, Taizō (1941) Haikai shichibushū, Tokio, Meiji Shoin. En línea: http:// www.j-texts.com/kinsei/sarumino.html. Último acceso el 12 de junio de 2020.

- (1979), Ebara Taizō Chosakushū VII, Tokio, Chūō Kōronsha. 
IsNARDI, Graciela (1979), «El hacedor de milagros. Octavio Paz, maestro de traductores», en Cuadernos Hispanoamericanos, 343-345. págs. 720-731. En línea: http://bibliotecanacionaldigital.gob.cl/colecciones/BND/00/ RC/RC0230512.pdf. Último acceso el 12 de junio de 2020.

Hayashiya, Eikichi (2005), «Recuerdos de Octavio Paz y sus sendas de Oku», en Revista de la Universidad de México, 19. págs. 84-88. En línea: https:// dialnet.unirioja.es/servlet/articulo?codigo=2930075. Último acceso el 12 de junio de 2020.

KeEne, Donald (1955), Anthology of Japanese Literature, Nueva York, Grove Press.

Miner, Earl (1969), Japanese Poetic Diaries, Los Ángeles, Universidad de California.

Min, Yong-Tae (1979), «Haiku en la poesía de Octavio Paz», en Cuadernos Hispanoamericanos, 343-345. págs. 696-707. En línea: http://www. bibliotecanacionaldigital.gob.cl/visor/BND:343110. Último acceso el 12 de junio de 2020. 